\title{
Plötzlicher Sehverlust: Warum der Ultraschall in der Primärdiagnostik ein wichtiges Mittel der Wahl sein sollte
}

Es kann von einer Sekunde auf die andere passieren: Die Sicht wird unscharf was gerade noch klar erkennbar war, scheint plötzlich zu verschwimmen, alles wirkt wie in „Nebel gehüllt“. Wer solche Symptome hat, leidet unter einem plötzlichen Sehverlust. Experten der DEGUM empfehlen in diesen Fällen den Ultraschall als wichtiges Diagnoseinstrument. Mithilfe der Sonografie können die häufigsten Ursachen eines akuten Sehverlusts - wie beispielsweise eine Netzhautablösung oder Verletzungen im Auge - besonders exakt und gesundheitsschonend dargestellt werden.

Ein plötzlicher Sehverlust kann beispielsweise auftreten, wenn das Auge verletzt wird etwa, wenn bei Heimwerkarbeiten kleine Fremdkörper hineingelangen. Auch ein akuter Arterienverschluss im Auge, wodurch die Durchblutung gestört wird, kann zu einem sofortigen Verlust des Sehvermögens führen. Als weitere Ursache sind sogenannte „Glaskörperblutungen“ im Auge zu nennen, die durch blutverdünnende Medikamente und/oder beispielsweise bei Diabetikern durch vermehrte Glaskörperzugwirkung (Glaskörperstränge) auf die Netzhaut auftreten. Gerade wenn der Einblick in das Augeninnere durch eine schlecht erweiterbare Blende (Pupille, Öffnung in der Regenbogenhaut/Iris) erschwert ist, kann der Augenultraschall hilfreich sein. Auch eine akute Ablösung der Netzhaut kann Ursache eines Visusverlusts sein.
Wer einen plötzlichen Verlust seiner Sehkraft bei sich oder einem Angehörigen bemerkt, sollte sich sofort in augenärztliche Behandlung begeben - denn im schlimmsten Fall kann es ohne Therapie zur dauerhaften Erblindung kommen. Die DEGUM empfiehlt, bei der augenärztlichen Untersuchung nach einem plötzlichen Visusverlust die Sonografie in die primäre Diagnostik einzubeziehen: „Moderne Ultraschalltechniken bieten mittlerweile ein gutes Auflösungsvermögen, in der Ultraschallbiomikroskopie können sogar kleinste rote Blutkörperchen im Auge dargestellt werden. Dabei ermöglicht die Sonografie eine viel genauere Darstellung als andere bildgebende Verfahren wie die Computertomografie (CT)“, betont Professor Dr. med. Frank Tost, Leiter der DEGUM-Sektion Ophthalmologie.

Ein weiterer Vorteil ist, dass mit der Sonografie eine dynamische Untersuchung des Auges möglich wird: „Während der Untersuchung kann der Patient sein Auge bewegen, was wichtige zusätzliche Informationen liefert, bei einer Computertomografie aber störend wäre“, sagt Tost, Oberarzt an der Universitätsmedizin Greifswald in der Klinik und Poliklinik für Augenheilkunde. „So können auch verstecktere Bereiche etwa im toten Winkel des Augenhinterabschnitts - ziemlich genau dargestellt werden." Neben der exakten Darstellung ist ein weiterer Vorteil, dass die Augenultraschalluntersuchung unkompliziert und sehr gesundheitsschonend erfolgt, da es keine Strahlenbelastung gibt.
Ursachen des plötzlichen Sehverlusts können auch Durchblutungsstörungen der Sehrinde und Entzündungen der hirnversorgenden Gefäße einschließlich des Auges sein. Hier werden dringlich interdisziplinäre Ultraschalluntersuchungen der Kopf- und Halsgefäße und des Herzens empfohlen.

Bei der Untersuchung untersucht der Arzt schonend durch das Augenlid oder er versetzt die Augenoberfläche zunächst in eine örtliche Betäubung. Anschließend begutachtet er es systematisch unter dem Ultraschall. Für den Augenultraschall stehen den Ärzten verschiedene Sonden mit Frequenzen zwischen 10 und 60 Megahertz zur Verfügung Die Sonden unterscheiden sich hinsichtlich ihrer Eindringtiefe, dem Auflösungsvermögen und der Abbildungsgeometrie.

Damit Augenärzte die Diagnose korrekt stellen können, ist eine adäquate fachliche Qualifikation eine wichtige Voraussetzung. „Wir empfehlen Augenärzten in einer Praxis eine Zertifizierung nach der DEGUM-Stufe I und solchen in einer spezialisierten Augenklinik ein Zertifikat der DEGUM-Stufe II“, sagt PD Dr. med. Ulrich Fries, Stellvertretender Leiter der DEGUM-Sektion Ophthalmologie und Chefarzt in der Augenklinik der Evangelischen Kliniken - JohanniterKrankenhaus in Bonn. Die DEGUM bietet regelmäßig Weiterbildungen an, mit denen Augenärzte diese Zertifizierungen erwerben können. 